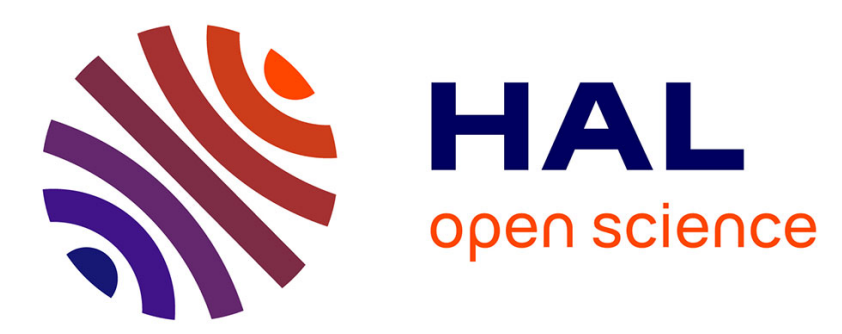

\title{
Using perturbatively selected configuration interaction in quantum Monte Carlo calculations
}

\author{
Emmanuel Giner, Anthony Scemama, Michel Caffarel
}

\section{To cite this version:}

Emmanuel Giner, Anthony Scemama, Michel Caffarel. Using perturbatively selected configuration interaction in quantum Monte Carlo calculations. Canadian Journal of Chemistry, 2013, 91 (9), pp.879-885. 10.1139/cjc-2013-0017 . hal-00992090

\section{HAL Id: hal-00992090 https://hal.science/hal-00992090}

Submitted on 16 May 2014

HAL is a multi-disciplinary open access archive for the deposit and dissemination of scientific research documents, whether they are published or not. The documents may come from teaching and research institutions in France or abroad, or from public or private research centers.
L'archive ouverte pluridisciplinaire HAL, est destinée au dépôt et à la diffusion de documents scientifiques de niveau recherche, publiés ou non, émanant des établissements d'enseignement et de recherche français ou étrangers, des laboratoires publics ou privés. 


\title{
Using perturbatively selected configuration interaction in quantum Monte Carlo calculations
}

\author{
Emmanuel Giner, Anthony Scemama, and Michel Caffarel \\ Lab. Chimie et Physique Quantiques, \\ CNRS-Université de Toulouse, France.
}

\begin{abstract}
Defining accurate and compact trial wavefunctions leading to small statistical and fixed-node errors in quantum Monte Carlo (QMC) calculations is still a challenging problem. Here, we propose to make use of selected configuration interaction (CI) expansions obtained by selecting the most important determinants through a perturbative criterion. A major advantage with respect to truncated CASSCF wavefunctions or CI expansions limited to a maximum number of excitations (e.g, CISD) is that much smaller expansions can be considered (many unessential determinants are avoided), an important practical point for efficient QMC calculations. The most important determinants entering first during the selection process (hierarchical construction) the main features of the nodal structure of the wavefunction can be expected to be obtained with a moderate number of determinants. Thanks to this property, the delicate problem of optimizing in a Monte Carlo framework the numerous linear/nonlinear parameters of the determinantal part of the trial wavefunction could be avoided. As a first numerical example, the calculation of the ground-state energy of the oxygen atom is presented. The best DMC value reported so far is obtained.

Keywords: Perturbatively Selected Configuration Interaction; quantum Monte Carlo; Fixed-Node Diffusion Monte Carlo
\end{abstract}




\section{GRAPHICAL ABSTRACT}

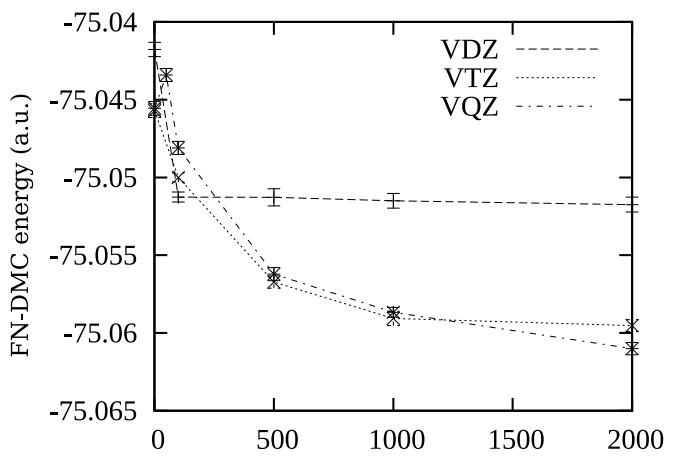

Number of determinants in the reference wave function 


\section{INTRODUCTION}

Quantum Monte Carlo (QMC) methods are known to be powerful stochastic approaches for obtaining accurate ground-state properties of quantum systems. For bosonic systems the results obtained are essentially exact, up to some statistical error as in any Monte Carlo approach (see, for example the reference simulations for the $\mathrm{He}_{4}$ quantum liquid[1]). In contrast, for Fermi systems where the antisymmetry of the wavefunction is to be imposed, the situation is different. As known, it has not been possible so far to devise a QMC algorithm for fermions that would be both stable (controlled fluctuations of the wavefunction sign) and exact (no systematic error in the limit of infinite simulation time). In practice, this difficulty known as the "fermionic sign problem" is circumvented by using the so-called "Fixed-Node" (FN) approach where the sign instability is removed at the expense of a small systematic (fixed-node) error. In short, the FN approach consists in defining a juxtaposition of bosonic-type simulations defined independently within each nodal pocket (domains of constant sign) of an approximate Fermi (antisymmetric) trial wavefunction, $\Psi_{T}$. When the nodes (or zeroes) of $\psi_{T}$ coincide with the exact nodes, the algorithm is exact. If not -which is the general case except for some elementary systems- a fixed-node error is introduced.[2] Using standard trial wavefunctions, this error is in general small, [3] typically a few percent of the correlation energy for total energies. However, this remarkable accuracy on total energies can still be insufficient when calculating the energy differences at the heart of quantitative chemistry:[4] Binding energies, energy variations along a reaction path, forces (viewed as infinitesimal differences), electronic affinities, electronic transition energies, etc. Indeed, on the total energy scale these differences are extremely small, at least of the same order of magnitude, and most often smaller, than the already very small fixed-node error. The precision on energy differences is thus directly related to the quality of the nodal hypersurfaces of the trial wavefunction and on the way the fixed-node error compensates or not when computing differences of large total energies.[5],[6] For this reason, defining trial wavefunctions with accurate nodes is still one of the important issues of quantum Monte Carlo (QMC) approaches for chemistry.

Besides this important aspect, the trial wavefunction is also directly related to the efficiency of QMC simulations (defined here as a good convergence of the estimators as a function of the simulation time and a low level of statistical fluctuations). The QMC meth- 
ods being highly CPU-intensive ("number crunching" approaches) this aspect is of particular importance. In the important case of the energy, the crucial role of the trial wavefunction is directly seen from the fact that the closest from the exact wavefunction the trial wavefunction is, the smallest the statistical error on the total energy is (zero-variance property, see e.g. [3]).

In view of the importance of the trial wavefunction, much effort has been made to propose and optimize functional forms that lead to accurate nodes and low level of fluctuations. In addition, the trial wavefunction must be compact enough to be rapidly evaluated at each of the millions and more Monte Carlo steps. The standard expression employed in QMC is the Jastrow-Slater form expressed as a short expansion over a set of Slater determinants multiplied by a global Jastrow factor describing explicitly the electron-electron and electron-electron-nucleus interactions and, in particular, imposing the electron-electron CUSP conditions associated with the zero-interelectronic distance limit of the true wavefunction. A variety of alternative forms aiming at better describing the exact wavefunction have been introduced. Without entering into the details, let us cite the geminal wavefunction of Sorella and coworkers,[7] the Pfaffian wavefunction of Mitáš and collaborators,[8], the backflow trial wavefunction of Rios et al.[9], the generalized valence bond (GVB) of Anderson and Goddard[10], the linear scaling GVB version of Fracchia et al.[11], the Jastrowvalence-bond wavefunction of Braida et al.[12], or the multi-Jastrow trial wavefunction of Bouabça et al.[13] Once a trial wavefunction has been chosen, its various parameters (Jastrow parameters, determinantal coefficients, molecular orbital coefficients, basis set exponents, and so on) are in general optimized. The criterion employed can be either the minimization of the variational energy associated with the trial wavefunction, $\left\langle\Psi_{T}|H| \Psi_{T}\right\rangle$, its variance $\left\langle\Psi_{T}\left|H^{2}\right| \Psi_{T}\right\rangle-\left\langle\Psi_{T}|H| \Psi_{T}\right\rangle^{2}$, or a combination of both. Note that optimizing hundreds/thousands of linear and nonlinear parameters within a framework where the energy and variance are subject to a statistical noise is not an easy task. A number of solutions have been proposed, let us just mention here the recent approach of Umrigar and collaborators[14] and references therein.

In this work we propose to exploit the multi-determinant expansions of the postHF approaches of quantum chemistry. So far, multi-determinant expansions in QMC have been mainly limited to the use of CASSCF-like or CI-like wavefunctions truncated at a moderate number of determinants or configurations (say, a maximum of a 
few thousands) selected by using some threshold in the expansion coefficients (see, e.g. $[15],[16],[17],[18],[19],[20],[11],[21])$. To the best of our knowledge, the only DMC calculation using a complete full CI wavefunction (expansion over all possible determinants in a given set of molecular orbitals) has been made in Ref. [22] for the $\mathrm{Li}_{2}$ molecule. However, this calculation involving about 16000 determinants has been possible only because the number of active electrons for such a molecule is very small. As well-known, due to the exponential increase of the dimension of the FCI space, to consider much larger systems is not realistic. Here, we propose to use trial wavefunctions based on truncated expansions containing the most "important" determinants while remaining compact enough to be evaluated at each Monte Carlo step (say, expansions involving at most a few hundreds of thousands of determinants). In quantum chemistry there is a long history of developing approximate schemes based on various approximations: Configuration Interaction expansion truncated to a certain level of excitation (single: CIS, double: CISD, etc.), exponential ansatz: CCSD, CCSD(T) etc., perturbative approaches: Møller-Plesset MP2, etc., CASSCF approaches, and so on. However, such schemes still generate too many determinants to be tractable in QMC and, furthermore, the choice of the criterion employed for truncation may be questionable. Here, we shall follow a different route introduced in the last decades by a number of authors (see, e.g., [23-31]). In a few words, the approach consists in building the multi-determinantal expansion iteratively by selecting at each step one determinant (or a group of determinants) according to a perturbative criterion. In short, a determinant $D_{i}$ (or a group of determinants) is added to the current wavefunction if its (their) energetic contribution(s) is (are) sufficiently large. In this work the formalism employed is close to the one adopted in the CIPSI algorithm.[24, 30] Finally, let us note that some time ago Koch and Dalgaard[32] proposed a method having some similar aspects (add one determinant at a time and try to keep the multi-determinant expansion as compact as possible) but resting on a completely different approach for building the expansion.

The contents of this paper is as follows. In Section II, the algorithm used for building the selected configuration interaction expansion (CIPSI-like algorithm) is presented. In Section III a few words about the Fixed-Node Diffusion Monte Carlo method employed here are given. In Section IV the first application to the calculation of the ${ }^{3} P$ ground-state energy of the oxygen atom is presented. The various aspects related to the implementation of the CIPSI algorithm and the fixed-node calculations are described. Finally, the main ideas and 
results of this work are summarized in Sec. V

\section{PERTURBATIVELY SELECTED CONFIGURATION INTERACTION}

In multi-determinantal expansions the ground-state wavefunction $\left|\Psi_{0}\right\rangle$ is written as a linear combination of Slater determinants $\left\{\left|D_{i}\right\rangle\right\}$, each determinant corresponding to a given occupation by the $N_{\alpha}$ and $N_{\beta}$ electrons of $N=N_{\alpha}+N_{\beta}$ orbitals among a set of $M$ spinorbitals $\left\{\phi_{1}, \ldots, \phi_{M}\right\}$ (restricted case). When no symmetries are considered the maximum number of such determinants is $\left(\begin{array}{c}M \\ N_{\alpha}\end{array}\right)\left(\begin{array}{c}M \\ N_{\beta}\end{array}\right)$, a number that grows factorially with $M$ and $N$. The best representation of the exact wavefunction in the determinantal basis is the Full Configuration Interaction (FCI) wave function written as

$$
\left|\Psi_{0}\right\rangle=\sum_{i} c_{i}\left|D_{i}\right\rangle
$$

where $c_{i}$ are the ground-state coefficients obtained by diagonalizing the matrix, $H_{i j}=\left\langle D_{i}|H| D_{j}\right\rangle$, within the full orthonormalized set, $\left\langle D_{i} \mid D_{j}\right\rangle=\delta_{i j}$, of determinants $\left|D_{i}\right\rangle$.

In its simplest form, our multi-determinant wavefunction is iteratively built as follows:

- Step 0: Start from a given determinant (e.g. the Hartree-Fock determinant) or set of determinants, thus defining an initial reference subspace: $S_{0}=\left\{\left|D_{0}\right\rangle, \ldots\right\}$. Diagonalize $H$ within $S_{0}$ and get the ground-state energy $E_{0}^{(0)}$ and eigenvector:

$$
\left|\Psi_{0}^{(0)}\right\rangle=\sum_{i \in S_{0}} c_{i}^{(0)}\left|D_{i}\right\rangle
$$

Here and in what follows, a superscript on various quantities is used to indicate the iteration number.

Then, do iteratively $(n=0, \ldots)$ :

- Step 1: Collect all different determinants $\left|D_{i_{c}}\right\rangle$ connected by $H$ to $\left|\Psi_{0}^{(n)}\right\rangle$, namely

$$
\left\langle\Psi_{0}^{(n)}|H| D_{i_{c}}\right\rangle \neq 0
$$


- Step 2: Compute the second-order perturbative energetic change of the total energy resulting from each connected determinant:

$$
\delta e\left(\left|D_{i_{c}}\right\rangle\right)=-\frac{\left\langle\Psi_{0}^{(n)}|H| D_{i_{c}}\right\rangle^{2}}{\left\langle D_{i_{c}}|H| D_{i_{c}}\right\rangle-E_{0}^{(n)}}
$$

- Step 3: Add the determinant $\left|D_{i_{c}^{*}}\right\rangle$ associated with the largest $|\delta e|$ to the reference subspace:

$$
S_{n} \rightarrow S_{n+1}=S_{n} \cup\left\{\left|D_{i_{c}^{*}}\right\rangle\right\}
$$

- Step 4: Diagonalize $H$ within $S_{n+1}$ to get:

$$
\left|\Psi_{0}^{(n+1)}\right\rangle=\sum_{i \in S_{n+1}} c_{i}^{(n+1)}\left|D_{i}\right\rangle \quad \text { with } \quad E_{0}^{(n+1)}
$$

- Go to step 1 or stop if the target size for the reference subspace has been reached.

Denoting $N_{\text {dets }}$ the final number of determinants in $\left|\Psi_{0}\left(N_{\text {dets }}\right)\right\rangle$ and $E_{0}\left(N_{\text {dets }}\right)$ the final energy, a perturbative second-order estimate of the exact energy -the so-called CIPSI energycan be obtained as

$$
E_{0}(\mathrm{CIPSI})=E_{0}\left(N_{\mathrm{dets}}\right)-\sum_{i \in M} \frac{\left\langle\Psi_{0}\left(N_{\mathrm{dets}}\right)|H| D_{i}\right\rangle^{2}}{\left\langle D_{i}|H| D_{i}\right\rangle-E_{0}\left(N_{\mathrm{dets}}\right)}
$$

where $M$ denotes the set of all determinants not belonging to the reference space and connected to the reference vector $\left|\Psi_{0}\left(N_{\text {dets }}\right)\right\rangle$ by $H$ (single and double excitations). In what follows the wave function $\left|\Psi_{0}\left(N_{\text {dets }}\right)\right\rangle$ that will be used for QMC calcaultations will be referred to as the reference wave function.

At this point a number of remarks are in order:

i.) Although the selection scheme is presented here for computing the ground-state eigenvector only, no special difficulties arise when generalizing the scheme to a finite number of states (see, e.g.[30]) 
ii.) The decomposition of the Hamiltonian $H$ underlying the perturbative second-order expression introduced in step 2 is given by

$$
H=H_{0}+\left\langle D_{i_{c}}|H| D_{i_{c}}\right\rangle\left|D_{i_{c}}\right\rangle\left\langle D_{i_{c}}\right|
$$

where $H_{0}$ is the restriction of $H$ to the reference subspace. This decomposition known as the Epstein-Nesbet partition[33, 34] is not unique, other possible choices are the Møller-Plesset partition[35] or the barycentric one,[24] see discussion in [30].

iii.) Instead of calculating the energetic change perturbatively, expression (4), it can be preferable to employ the non-perturbative expression resulting from the diagonalization of $H$ into the two-dimensional basis consisting of the vectors $\left|\Psi_{0}^{(n)}\right\rangle$ and $\left|D_{i_{c}}\right\rangle$. Simple algebra shows that the energetic change is given by

$$
\delta e\left(\left|D_{i_{c}}\right\rangle\right)=\frac{\left[\left\langle D_{i_{c}}|H| D_{i_{c}}\right\rangle-E_{0}\left(N_{\text {dets }}\right)\right]\left[1-\sqrt{\left.1+\frac{4\left\langle\Psi_{0}^{(n)}|H| D_{i_{c}}\right\rangle^{2}}{\left[\left\langle D_{i_{c}}|H| D_{i_{c}}\right\rangle-E_{0}\left(N_{\text {dets }}\right)\right]^{2}}\right]}\right.}{2}
$$

In the limit of small transition matrix elements, $\left\langle\Psi_{0}^{(n)}|H| D_{i_{c}}\right\rangle$, both expressions (4) and (7) coincide. In what follows the non-perturbative formula will be used.

iv.) In step 3 a unique determinant is added at each iteration. Adding a few of them simultaneously is also possible, a feature particularly desirable when quasi-degenerate low-lying determinants are showing up. In the applications to follow this possibility has been systematically used by keeping at each iteration all determinants associated with an energetic change whose absolute value is greater than a given threshold.

v.) The implementation of this algorithm can be performed using limited amount of central memory. On the other hand, the CPU time required is essentially proportional to $N_{\text {dets }} N_{\text {occ }}^{2} N_{\text {virt }}^{2}$ where $N_{\text {occ }}$ is the number of occupied molecular orbitals and $N_{\text {virt }}$ the number of virtual orbitals. 


\section{THE FIXED-NODE DIFFUSION MONTE CARLO}

In this work the Fixed-Node Diffusion Monte Carlo (FN-DMC) method -the standard quantum Monte Carlo electronic-structure approach for molecules- is employed. For a detailed presentation of its theoretical and practical aspects, the reader is referred to the literature, e.g [36-38]. Here, we just recall that the central quantity of such approaches is the trial wavefunction $\Psi_{T}$ determining both the quality of the statistical convergence (good trial wavefunctions $=$ small statistical fluctuations) and the magnitude of the fixed-node bias resulting from the approximate nodes of the trial wavefunction. The computational cost of FN-DMC is almost entirely determined by the evaluation at each Monte Carlo step of the value of $\Psi_{T}$ and its first (drift vector) and second derivatives (Laplacian needed for the local energy). In view of the very large number of MC steps usually required (typically at least millions and often much more) to be able of computing such quantities very rapidly is essential. In contrast with most implementations of FN-DMC where compact forms for $\Psi_{T}$ are used (typically, at most a few hundreds of determinants) quite lengthy multi-determinantal expansions are considered here (up to 200000 determinants in the numerical application presented below). As a consequence, some care is required when computing such expansions. At first glance, the CPU cost is expected to be proportional to the number of determinants $N_{\text {dets }}$ involved in the expansion of the trial wavefunction. Actually, it is not true since in the spin-free formalism used in QMC (Ref. [39] and also [36-38]) each Slater determinant expressed in terms of spin-orbitals decomposes into a product of two determinants, each of them corresponding to a given occupation of a set of purely spatial molecular orbitals. The number of different determinants to be computed is thus of the order of $\sqrt{N_{\text {dets }}}$ and not $N_{\text {dets }}$. Another point having a significant impact on the computational cost is the order with which determinants are calculated. Indeed, avoiding to re-compute successive determinants differing from preceding ones only by a single or double molecular orbital substitution can be efficient. In practice, this is done by re-actualizing the determinants using a Sherman-Morisson-type formula.[40] Now, to determine which ordering is the most effective is a difficult problem of combinatorial complexity. Here, we employ a simple strategy based on a preliminary preconditioning step. More involved strategies for treating bigger systems and larger expansions will be presented elsewhere. 


\section{NUMERICAL RESULTS}

In this section CIPSI and FN-DMC calculations of the ${ }^{3} P$ ground-state energy of the oxygen atom are presented. Among the first-row atoms $\mathrm{O}$ is known to be the atom whose nodes are the most difficult to describe (fixed-node error of about $6 \%$ of the correlation energy when using Hartree-Fock nodes[41]).

\section{A. CIPSI wavefunctions}

Selected configuration interation wave functions are constructed using Hartree-Fock canonical atomic orbitals obtained with various Dunning basis sets, [42] cc-pVXZ (denoted as VXZ), aug-cc-pVXZ (denoted as AVXZ), and cc-pCVXZ basis sets (denoted as CVXZ) with $\mathrm{X}=\mathrm{D}, \mathrm{V}, \mathrm{T}, \mathrm{Q}, 5$. All-electron calculations are performed and the full set of atomic orbitals are active in the excitation process, except when freezing the $1 s$ innermost atomic orbital as indicated (FC for Frozen Core).

In figure 1 the convergence of the variational $\mathrm{E}_{0}\left(\mathrm{~N}_{\text {dets }}\right)$ and CIPSI energies (variational + PT2 correction) for the VDZ, VTZ, VQZ, and V5Z basis sets as a function of the number of determinants kept in the selection process is shown. The exact non-relativistic ground-state energy -75.0674 evaluated by Chakravorty et al. [43] is also indicated. As seen a striking feature is the extremely rapid convergence of total energies. To better visualize this convergence, particularly at small number of determinants, the abscissa has been scaled logarithmically. As it should be the variational energy associated with the reference wavefunction converges to the asymptotic limit from above. In contrast, the CIPSI energy including second-order correction is found to systematically converge from below. Remarkably, in all cases the convergence is attained for a tiny fraction of the total number of determinants of the FCI space. Quantitatively, it is interesting to determine, for each basis set, the minimum number of determinants needed to reach one percent or less of the total correlation energy computed within the basis considered. For the DZ basis set this level of accuracy is attained with about 500 determinants for $\mathrm{E}_{0}\left(\mathrm{~N}_{\text {dets }}\right)$ and about 80 determinants for the CIPSI energy. For TZ, QZ, and 5Z, these numbers are $(\sim 10000, \sim 40),(\sim 30000, \sim 90)$, and $(\sim 50000, \sim$ 300), respectively. It is seen that the CIPSI energy convergence depends weakly on the basis set size, a remarkable result illustrating that the second-order correction is able to recover 
most of the missing part. In contrast, for the variational energy the minimum number of determinants needed increases as a function of the basis set size. However, this increase is moderate when compared with the exponential increase of the FCI space dimensionality. Quantitatively, the target accuracy of less than $1 \%$ of the correlation energy is attained at the variational level for a fraction of determinants given by $f=\frac{N_{\text {dets }}}{N_{\mathrm{FCI}}} \sim 7.010^{-4}, \sim 2.010^{-5}$, $\sim 3.310^{-7}$, and $\sim 9.110^{-9}$ for the DZ, TZ, QZ, and $5 \mathrm{Z}$ basis sets, respectively. However, note that in the context of building compact trial wave functions for QMC the key quantity is the total number of contributing determinants (associated with the computational cost) and not the fraction. To better visualize how the energies converge, Figure 2 presents a blow-up of figure 1 in the region of the large number of determinants for the two biggest basis sets, QZ and 5Z. Very similar behaviors are obtained for the DZ and TZ smaller basis sets. Once more the very rapid convergence of the full energy including the second-order energy correction is clearly seen. Regarding the variational energy, the convergence is much less impressive but still very satisfactory. It is reasonable to expect the convergence of both variational and CIPSI energies to a common asymptotic value as it should be.

A key aspect of CIPSI is that the multi-determinantal expansion is built by selecting the determinants according to their contribution to the wavefunction and total energy and not according to some fixed maximum level of excitations (single, double, triple,..) as it is usual in most post-Hartree-Fock schemes. In figure 3 this aspect is illustrated by showing the evolution of the number of selected determinants corresponding to a given excitation level as a function of the total number of determinants kept during the selection process up to the first 50000 determinants (TZ basis basis set). Starting from the unique HF determinant with no excitation (determinant \#1), the first determinants to enter are of double-excitation type (from determinant \#2 to determinant \#19), the 20-th is a single excitation determinant, etc. Table I reports when a determinant of a given excitation level class appears for the first time ("occurence" time) together with the total number of determinants in each class of excitation level at the end of the selection process. It is remarkable to see how far we are from a selection process where the derminants would be chosen according to their level of excitation (lower numbers of excitations first). In particular, high-level excitations enter quite soon into the wavefunction: At $N=1188$ and $N=8159$ for 5-uple and 6-uple excitations, respectively (here, no 7- and 8-uple excitations are observed). Single and double-excitation are found to saturate while the other types of excitations are still increasing in number. After 50 
000 selected determinants, the most numerous determinants are of quadruple- and tripleexcitation types.

Table II collects the set of results obtained with various basis sets and truncation levels (number of determinants kept in the reference wavefunction). The data are presented for the VDZ, AVDZ, VTZ, AVTZ, VQZ, AVQZ, CVQZ, and V5Z basis sets. In the case of the VDZ, VTZ, and VQZ basis set results obtained by freezing the 1s core orbital are also given. When available (VDZ and VTZ basis sets) the Full CI (FCI) energy is indicated. We also report the FCI-QMC results of Booth and Alavi[44] for the AVDZ, AVTZ, AVQZ, and AV5Z basis sets. In each case the total number of determinants of the FCI space is given. For each total energy the percentage of the exact correlation energy is given in brackets. As already seen qualitatively on the figures 1 and 2 the convergence of the variational and CIPSI energies to the FCI limit is very statisfactory for all basis sets. For the DZ basis set, the FCI, variational, and CIPSI energies coincide with seven digits in the VDZ-FC and VDZ cases and with about six digits for AVDZ. For the larger basis sets, the differences are always smaller than the milliHartree (chemical accuracy). Finally, it is interesting to note that using a basis set adapted to the core region (CVQZ) an energy value of -75.054 38 has been obtained here, much lower than the best value of Booth and Alavi obtained with the V5Z basis set, namely $\mathrm{E}_{0}=-75.037$ 49(6). This latter result illustrates the quantitative importance of the core contribution to the total atomic energy. However, when computing energy differences for molecular properties such a purely atomic contribution is expected to cancel almost entirely.

\section{B. QMC calculations using selected configuration interaction wavefunctions}

In figures 4 and 5 the fixed-node DMC ground-state energies for the oxygen atom as a function of the number of determinants kept for the VDZ, VTZ, and VQZ basis sets are presented. Figure 4 shows the entire curve using a logarithmic scale for the abscissa, while the region at small number of determinants is presented in figure 5 with a standard linear scale. As seen, except in one case that shall be discussed below (VQZ at $N_{\text {dets }}=50$ ), the fixednode energy is found to decrease regularly with the number of determinants. Such a result shows that a simple and systematic control of the nodal quality of the trial wavefunction through determinantal selection is possible. Although such a remarkable behavior is not 
easy to justify mathematically, it is coherent with the fact that the selection process realizes a hierarchical build up of the main features of the wavefunction and, thus, of its nodal structure. It is also coherent with the fact that the second-order perturbative correction was able to recover most of the missing part of the FCI energy very rapidly as a function of the number of determinants kept, thus confirming that the most important features enter quickly the reference wavefunction upon selection.

In most $\mathrm{QMC}$ works a prelimary optimization step of the trial wavefunction is performed before running DMC calculations. It is done because the introduction of the various $N$-body terms aiming at better describing the physical properties of the trial wavefunction (Jastrow factor, backflow transformation, geminal functions, etc.) has a strong impact on the initial determinantal part optimized in the absence of such terms. To decrease the fixed-node error, it is thus necessary to re-optimize all parameters of the trial wavefunction including those of the determinantal part (all molecular orbitals and determinantal coefficients). In practice, it can be a particularly tedious task, although much effort has been produced to make it as automatic as possible. The fact that the objective function to be minimized (total energy, energy variance, or a combination of both) is calculated with a statistical noise and that most parameters are non-linear are the two main difficulties for the optimization. Here, the situation is different since the optimization of the determinantal part is overcome when using perturbatively selected interaction configuration expansions. Eventually, the only optimizable parameters left are those not changing the nodes (typically the Jastrow parameters). Here, we have chosen to compute the FN-DMC energies using the pure CIPSI wave functions without Jastrow term, so that no optimization at all was necessary. Actually, the deterministic construction of a reference function through the diagonalization of a truncated Hamiltonian matrix must be considered as an optimization step (minimization of the total energy with respect to the coefficients of the multi-determinantal expansion). However, in sharp contrast with what happens in QMC, such a step is simple and automatic (no noise and linear parameters). This aspect will be particularly interesting when more complex systems will be considered. Finally, a few words of caution are in order. As seen above, in one case (VQZ basis set and small number of determinants, see Figs.4 and 5) the fixed-node energy is found to go up instead of decreasing. This result may indicate that the behavior of the fixed-node error is not so simple. However, it is also possible to interpret it as a transient effect related to the small number of determinants and large basis set regime. 
In Table III the Fixed-Node DMC energies obtained using several basis sets and various sizes of the reference wave function are presented. The correlation energy recovered in the FN-DMC calculations varies from $90 \%$ to nearly $100 \%$ depending on the nodal structure of the reference wave function. As was the case at the FCI level, to use a basis set adapted to the core region (CVQZ) is quantitatively important when highly-accurate total FN-DMC energies are searched for. This result illustrates the fact that the nodes in the nucleus region play a significant role. Our best total energy is obtained with the CVQZ basis set and 200 000 determinants. The value obtained is $-75.0658 \pm 0.0001$ recovering $99.4 \pm 0.1 \%$ of the correlation energy. To the best of our knowledge it is the best FN-DMC value reported so far for the oxygen atom. Note that it is slighter lower than the value of -75.065 $4 \pm$ 0.0001 obtained very recently by Seth et al.[20] with a fully optimized multideterminantJastrow-backflow trial wave function. For comparison Table III reports also some of the most accurate energies obtained for this atom by different methods. At the FCI level the best result we know is that of Booth and Alavi.[44] At the FN-DMC level, it is that of Seth et al. just mentioned. Finally, to the best of our knowledge the best energy reported up now is the value obtained by Gdanitz using the r12-MR-ACPF method.[45]

\section{SUMMARY}

In this work we have proposed to use trial wave functions for QMC based on selected configuration interaction (CI) expansions. The expansion is built iteratively by selecting at each step the most important determinants through a perturbative criterion. In short, a determinant $D_{i}$ (or a group of determinants) is added to the reference wavefunction if its (their) energetic contribution(s) is (are) sufficiently large (CIPSI-like algorithm). In practice, the expansion is stopped for the maximum number of determinants compatible with a rapid evaluation of the trial wavefunction at each step of the QMC calculation (here, a few hundreds of thousands of determinants for the oxygen atom). The main advantages of such wave functions are as follows.

i.) In constrast with truncated CASSCF wavefunctions or CI expansions limited to a maximum number of excitations (e.g, CISD), near FCI quality for the reference function can be reached with a much smaller number of determinants (many determinants with negligible weight in the exact wavefunction are avoided). For the case of the oxygen atom, the error 
with respect to the FCI limit for the DZ and TZ basis sets is systematically smaller than one milliHartree with a number of determinants not exceeding $10^{5}$. For the largest QZ and $5 \mathrm{Z}$ and a comparable number of determinants, the error does not exceed a few milliHartree.

ii.) Because the reference wave function is built hierarchically (most dominant determinants first), it is reasonable to expect that its overall quality improves as the number of determinants is increased and, in particular, its nodal structure. In this work, this point has been verified for the oxygen atom but its validity for more complex systems remains to be investigated.

iii.) Taking for granted the nodal quality of the multi-determinantal wavefunction when the CI expansion is sufficiently large, the tedious and delicate task of re-optimizing in a QMC framework the numerous linear and nonlinear parameters of the determinantal part can be avoided. Such a possibility of constructing optimal nodes in a purely deterministic and automatic way is a very appealing feature for future applications.

Finally, let us insist that the various aspects just discussed need to be investigated for more realistic systems beyond the oxygen atom. A study of the potential energy curve of the first-row diatomics molecules is presently underway.

Acknowledgments. One of us (MC) would like to dedicate this work with sincere admiration and friendship to Dennis Salahub who was very early interested in emerging quantum Monte Carlo techniques for computational chemistry.[46] We would also like to acknowledge interesting discussions with Nathalie Guihery and Jean-Paul Malrieu (Toulouse). AS and MC thank the Agence Nationale pour la Recherche (ANR) for support through Grant No ANR 2011 BS08 004 01. This work has been made through generous computational support from CALMIP (Toulouse) under the allocation 2012-0510, GENCI, and CCRT (CEA).

[1] Ceperley D.M. Rev. Mod. Phys. 199567279.

[2] Note that not all nodal pockets need to be considered -which would be an irrealistic task for large systems- thanks to a "tiling" theorem stating that all the nodal pockets of the fermionic ground-state are essentially equivalent and related by permutational invariance.[47]

[3] Foulkes, W.M.C.; Mitáš, L; Needs, R.J.; Rajagopal, G. Rev. Mod. Phys. 20017333. 
[4] This problem discussed here within the framework of QMC is of course present in any computational chemistry approach.

[5] Bouabça, T.; Caffarel, M.; N. Ben Amor, N.; Maynau, D. J. Chem. Phys. 2009130114107.

[6] Toulouse, J.; Caffarel, M.; Hoggan, P.E.; Reinhardt, P.; Umrigar, C.J. In Advances in the Theory of Quantum Systems in Chemistry and Physics Hoggan, P.E.E.; Brandas, E.J.J.; Maruani, J; Piecuch, P; Delgado-Barrio, G. (Eds) Progress in Theoretical Chemistry and Physics 20122255.

[7] Casula, M.; Attaccalite, C.; Sorella, S. J. Chem. Phys. 20041217110.

[8] Bajdich, M.; Mitáš, L.; Wagner, L.K.; Schmidt, K.E. Phys. Rev. B 200877115112.

[9] Lopez Rios, P.; Ma, A.; Drummond, N.D.; Towler, M.D., Needs, R.J. Phys. Rev. E 200674 066701.

[10] Anderson, A.G.; Goddard III, W.A. J. Chem. Phys. 2010132164110.

[11] Fracchia, F.; Filippi C.; Amovilli C. J. Chem. Theory Comput. 201281943.

[12] Braîda, B.; Toulouse J.; Caffarel M.; Umrigar C.J. J. Chem. Phys. 2011134084108.

[13] Bouabça, T.; Braîda, B.; Caffarel M. J. Chem. Phys. 2010133044111.

[14] Umrigar, C.J.; Toulouse, J.; Filippi, C.; Sorella, S.; Hennig, R.G. Phys. Rev. Lett. 200798 110201.

[15] Filippi, C.; Umrigar, C.J. J. Chem. Phys. 1996105213.

[16] Flad, H.J.; Caffarel M.; Savin, A. Quantum Monte Carlo calculations with multi-reference trial wave functions Recent Advances in Quantum Monte Carlo Methods 1997 World Scientific Publishing

[17] Caffarel, M.; Hernandez-Lamoneda, R.; Scemama, A.; Ramirez-Solis, A. Phys. Rev. Lett. 200799153001.

[18] Bajdich, M.; Tiago, M.L.; Hood, R.Q.; Kent, P.R.C.; Reboredo, F.A. Phys. Rev. Lett. 2010 104193001.

[19] Clark, B.K.; Morales, M.A.; McMinis, J.; Kim, J.; Scuseria, G.E. J. Chem. Phys. 2011135 244105.

[20] Seth, P.; Lopez Rios, P.; Needs, R.J. J. Chem. Phys. 2011134084105.

[21] Morales, M.A.; McMinis, J.; Clark, B.K.; Kim, J.; Scuseria, G.E. J. of Chem. Theory and Comput 201282181.

[22] Monari, A.; Scemama, A.; Caffarel, M. In Remote Instrumentation for eScience and Related 
Aspects 2012 Davoli, F.; Lawenda, M.; Meyer, N.; Pugliese, R.; Wglarz, J.; Zappatore, S. (Eds.), Springer New York, 195-207. http://dx.doi.org/10.1007/978-1-4614-0508-5_13

[23] Bender C.F.; Davidson E.R. Phys. Rev. 196918323.

[24] Huron, B.; Rancurel, P.; Malrieu, J.P. J. Chem. Phys. 1973585745.

[25] Buenker, R.J.; Peyerimholf, S.D. Theor. Chim. Acta 19743533.

[26] Buenker, R.J.; Peyerimholf, S.D. Theor. Chim. Acta 197539217.

[27] Buenker R.J.; Peyerimholf, S.D; Butscher, W. Mol. Phys. 197835771.

[28] Bruna, P.J.; Peyerimholf, S.D.; Buenker, R.J. Chem. Phys. Lett. 198072278.

[29] Buenker, R.J.; Peyerimholf, S.D.; Bruna, P.J. In Computational Theoretical Organic Chemistry 1981 Reidel, Dordrecht 55.

[30] Evangelisti, S.; Daudey, J.P.; Malrieu, J.P. Chem. Phys. 19837591.

[31] Harrison, R.J. J. Chem. Phys. 1991945021.

[32] Koch, H.; Dalgaard, E. Chem. Phys. Lett. 1993212193.

[33] Epstein, P.S. Phys. Rev. 192628695.

[34] Nesbet, R.K. Proc. Roy. Soc. 1955 A230 312.

[35] Møller, C.; Plesseti, M.S. Phys. Rev. 193446618.

[36] Hammond, B.L.; Lester Jr., W.A.; Reynolds, P.J. In Monte Carlo Methods in Ab Initio Quantum Chemistry, World Scientific Lecture and Course Notes in Chemistry Vol. 1, 19941 Singapore

[37] Towler, M.D. Quantum Monte Carlo, or, how to solve the many-particle Schrdinger equation accurately whilst retaining favourable scaling with system size, 2011 Wiley.

[38] Caffarel, M. Quantum Monte Carlo Methods in Chemistry, In Encyclopedia of Applied and Computational Mathematics 2012, Springer.

[39] Matsen, F.A. Adv. Quantum Chem. 196459.

[40] Press, W.H.; Flannery, B.P.; Teukolsky, S.A.; Vetterling, W.T. Numerical Recipes in C: The Art of Scientific Computing 1992, Cambridge University Press.

[41] Brown M.D.; Trail, J.R.; Lopez Rios, P; Needs, R.J. J. Chem. Phys. 2001126224110.

[42] Dunning, T.H. J. Chem. Phys. 1989901007.

[43] Chakravorty, S.J.; Gwaltney, S.R.; Davidson, E.R.; Parpia, F.A; Froese Fischer, C. Phys. Rev. A 1993473649.

[44] Booth, G.H.; Alavi, A. J. Chem. Phys. 2010132174104. 
[45] Gdanitz R.J. J. Chem. Phys. 19981099795.

[46] Caffarel, M.; Claverie, P.; Mijoule C.; Andzelm, J.; Salahub, D.R. J. Chem. Phys.1989 90 990.

[47] Ceperley, D.M. J. Stat. Phys. 1991631237. 


\begin{tabular}{|ccc|}
\hline Excitation class & First occurence & Total number of determinants \\
\hline \hline None $(\mathrm{HF})$ & 1 & 1 \\
Single & 20 & 34 \\
Double & 2 & 2205 \\
Triple & 194 & 16870 \\
Quadruple & 413 & 29618 \\
Quintuple & 1188 & 1184 \\
Sextuple & 8159 & 88 \\
Septuple & 0 & 0 \\
Octuple & 0 & 0 \\
\hline \hline Total & 50000 \\
\hline \hline
\end{tabular}

TABLE I: First occurence and total number of determinants of each class of excitation during the selection process up to the first 50000 determinants (VTZ basis set). 


\begin{tabular}{|c|c|c|c|c|c|c|}
\hline Basis set & $N_{\text {dets }}$ & $E_{0}(\mathrm{FCI})$ & $N_{\text {dets }}$ & $E_{0}\left(N_{\text {dets }}\right)$ & $E_{\mathrm{PT} 2}$ & $E_{0}(\mathrm{CIPSI})$ \\
\hline \multicolumn{7}{|l|}{ DZ } \\
\hline $\mathrm{VDZ} \mathrm{FC}^{b}$ & $\sim 5.610^{4}$ & -74.91015 & 50000 & $-74.91015[39.1 \%]$ & 0.0 & $-74.91015[39.1 \%]$ \\
\hline VDZ & $\sim 7.310^{5}$ & -74.91175 & 50000 & $-74.91175[39.7 \%]$ & $-3.510^{-9}$ & $-74.91175[39.7 \%]$ \\
\hline AVDZ & $\sim 5.910^{7}$ & $-74.92772(2)^{a}[45.9 \%]$ & 50000 & $-74.92767[45.9 \%]$ & $-9.910^{-6}$ & $-74.92768[45.9 \%]$ \\
\hline \multicolumn{7}{|l|}{$\mathrm{TZ}$} \\
\hline $\mathrm{VTZ} \mathrm{FC}^{b}$ & $\sim 9.610^{6}$ & -74.97424 & 50000 & $-74.97415[63.9 \%]$ & $-1.510^{-5}$ & $-74.97417[63.9 \%]$ \\
\hline VTZ & $\sim 5.810^{8}$ & $-74.98528 \quad[68.2 \%]$ & 100000 & $-74.98519[68.1 \%]$ & $-9.010^{-5}$ & $-74.98528[68.2 \%]$ \\
\hline AVTZ & $\sim 2.110^{10}$ & $-74.99077(4)^{a}[70.3 \%]$ & 100000 & $-74.99013[70.1 \%]$ & $-2.910^{-4}$ & $-74.99041[70.2 \%]$ \\
\hline \multicolumn{7}{|l|}{ QZ } \\
\hline $\mathrm{VQZ} \mathrm{FC}^{b}$ & $\sim 4.510^{8}$ & - & 50000 & $-74.99315[71.2 \%]$ & $-4.310^{-4}$ & $-74.99357[71.4 \%]$ \\
\hline VQZ & $\sim 9.110^{10}$ & - & 100000 & $-75.02269[82.7 \%]$ & $-3.210^{-4}$ & $-75.02300[82.8 \%]$ \\
\hline AVQZ & $\sim 2.010^{12}$ & $-75.02534(4)^{a}[83.7 \%]$ & 200000 & $-75.02269[82.7 \%]$ & $-2.110^{-3}$ & $-75.02476[83.5 \%]$ \\
\hline CVQZ & $\sim 2.710^{12}$ & - & 250000 & $-75.05167[93.9 \%]$ & $-2.710^{-3}$ & $-75.05438[95.0 \%]$ \\
\hline \multicolumn{7}{|l|}{$5 \mathrm{Z}$} \\
\hline V5Z & $\sim 5.610^{12}$ & - & 200000 & $-75.02740[84.5 \%]$ & $-9.910^{-4}$ & $-75.02839[84.9 \%]$ \\
\hline AV 5Z & $\sim 8.510^{13}$ & $-75.03749(6)^{a}[88.4 \%]$ & & & & \\
\hline
\end{tabular}

TABLE II: Variational total energy, $E_{0}\left(N_{\text {dets }}\right)$, and CIPSI total energy, $E_{0}(\mathrm{CIPSI})=E_{0}\left(N_{\text {dets }}\right)$ $+E_{\mathrm{PT} 2}$, for the ${ }^{3} \mathrm{P}$ ground-state of the oxygen atom using various basis sets and numbers of determinants in the reference wavefunction. For each energy the percentage of the exact correlation energy recovered is given in brackets. FCI-QMC calculations of Booth and Alavi[44] are given for comparison.

${ }^{a}$ Values from Ref.[44].

${ }^{b} \mathrm{FC}$ refers to a $\left(1 s^{2}\right)$ Frozen-Core. 


\begin{tabular}{|c|c|c|c|}
\hline Basis set & $N_{\text {dets }}$ & $E_{0}(\mathrm{FN}-\mathrm{DMC})$ & Correlation energy (\%) \\
\hline cc-pVDZ & $1(\mathrm{HF})$ & $-75.0418(5)$ & $90.1(2)$ \\
\hline cc-pVDZ & 5000 & $-75.0519(4)$ & $94.0(2)$ \\
\hline cc-pVTZ & $1(\mathrm{HF})$ & $-75.0457(4)$ & $91.6(2)$ \\
\hline cc-pVTZ & 2000 & $-75.0595(4)$ & $96.9(2)$ \\
\hline cc-pVQZ & 20000 & $-75.0642(2)$ & $98.8(1)$ \\
\hline cc-pCVQ2 & 100000 & $-75.0658(1)$ & $99.4(1)$ \\
\hline \multicolumn{4}{|c|}{ Other works } \\
\hline \multicolumn{2}{|c|}{ FCIQMC $^{a}$} & $-75.03749(6)$ & $88.40(2)$ \\
\hline \multicolumn{2}{|l|}{ FN-DMC ${ }^{b}$} & $-75.0654(1)$ & $99.2(1)$ \\
\hline \multicolumn{2}{|c|}{ r12-MR-ACPF ${ }^{c}$} & -75.066960 & 99.83 \\
\hline \multicolumn{2}{|c|}{ Exact $\mathrm{NR}^{d}$} & -75.0674 & 100.0 \\
\hline
\end{tabular}

TABLE III: FN-DMC energies and percentages of the correlation energy recovered for the ${ }^{3} P$ ground-state of the oxygen atom using various basis sets and truncation levels for the reference wavefunction. Comparison with accurate values available in the literature.

${ }^{a}$ Ref.[44]
${ }^{b}$ Ref.[20]
${ }^{c}$ Ref.[45]
${ }^{d}$ Ref.[43] 


\section{FIGURE CAPTIONS}

- Fig. 1 Convergence of the variational $\mathrm{E}_{0}\left(\mathrm{~N}_{\text {dets }}\right)$ (denoted VXZ) and CIPSI energies (variational + PT2 correction, denoted CIPS/VXZ) for the VDZ, VTZ, VQZ, and V5Z basis sets as a function of the number of determinants kept in the selection process. FCI values for the VDZ and VTZ basis sets are indicated together with the estimated exact non-relativistic ground-state energy[43].

- Fig. 2 Blow-up of figure 1 in the region of large number of determinants for the two biggest basis sets, VQZ and V5Z.

- Fig. 3 Number of determinants in each excitation class (single, double, triple, quadruple, quintuple, sextuple excitations) with respect to the total number of determinants in the reference wave function.

- Fig. 4 Fixed-node DMC ground-state energies for the oxygen atom as a function of the number of determinants kept for the VDZ, VTZ, and VQZ basis sets. A logarithmic scale for the abscissa is used.

- Fig. 5 Fixed-node DMC ground-state energies in the small number of determinants region. 


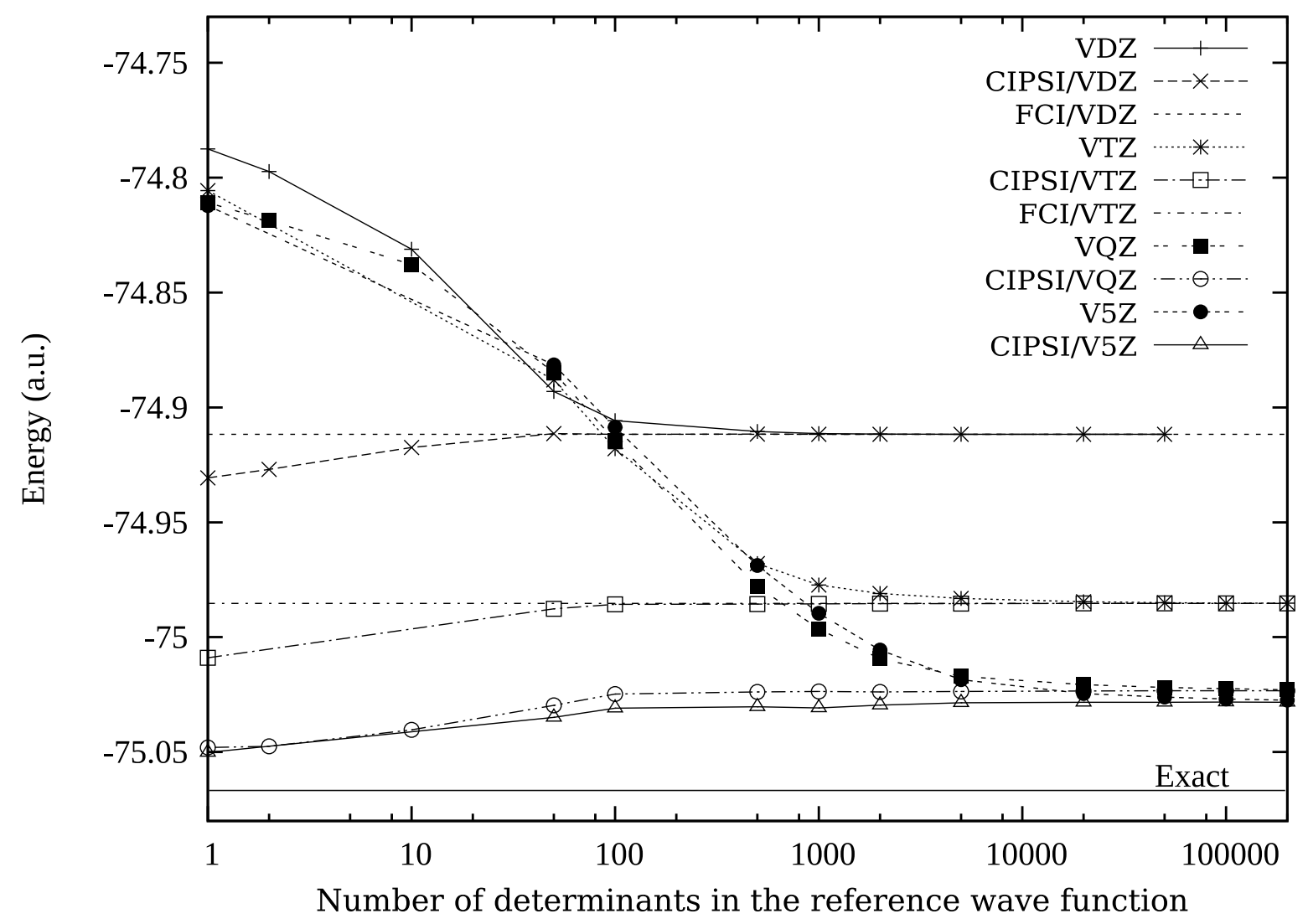

FIG. 1: 


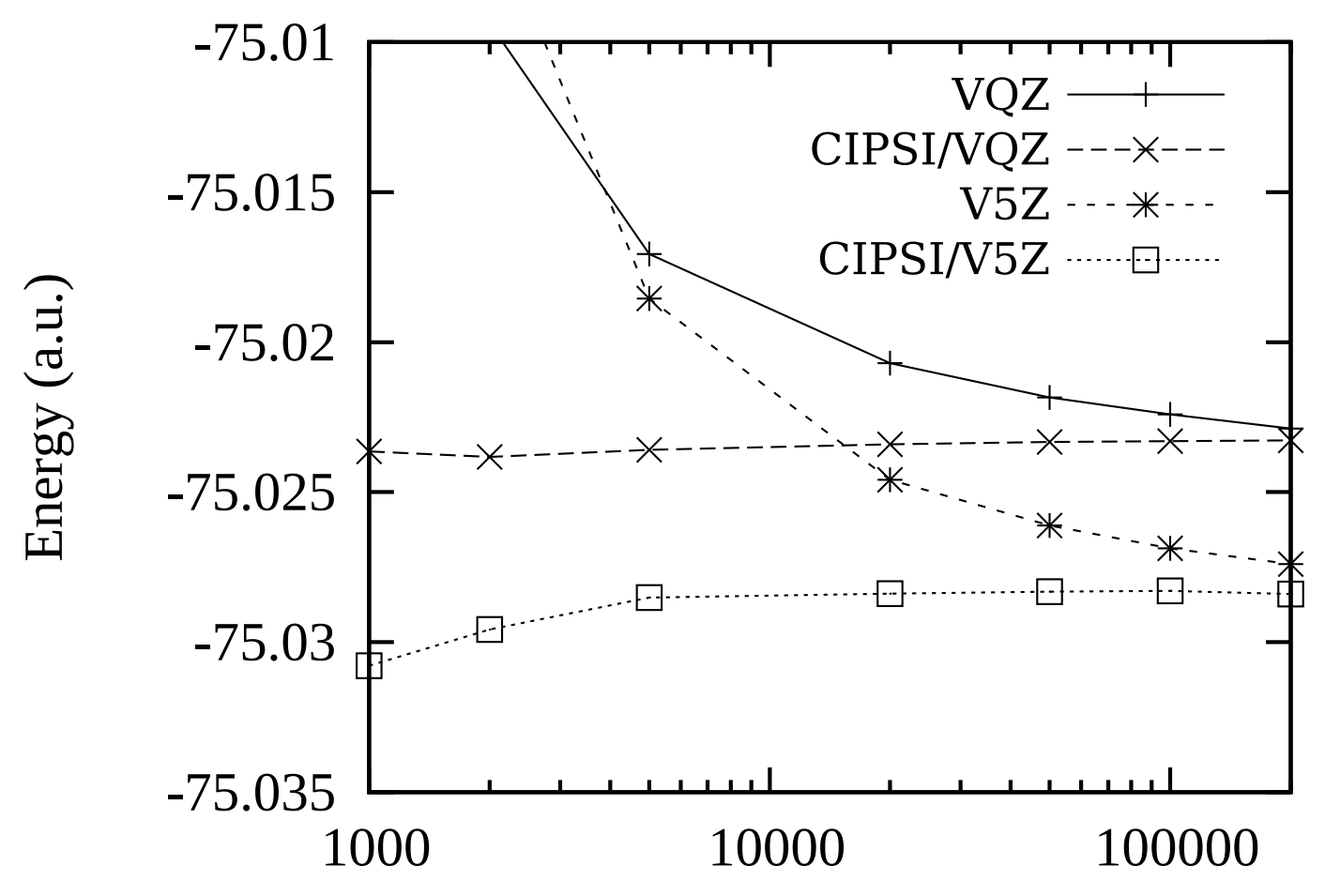

Number of determinants in the reference wave function

FIG. 2: 


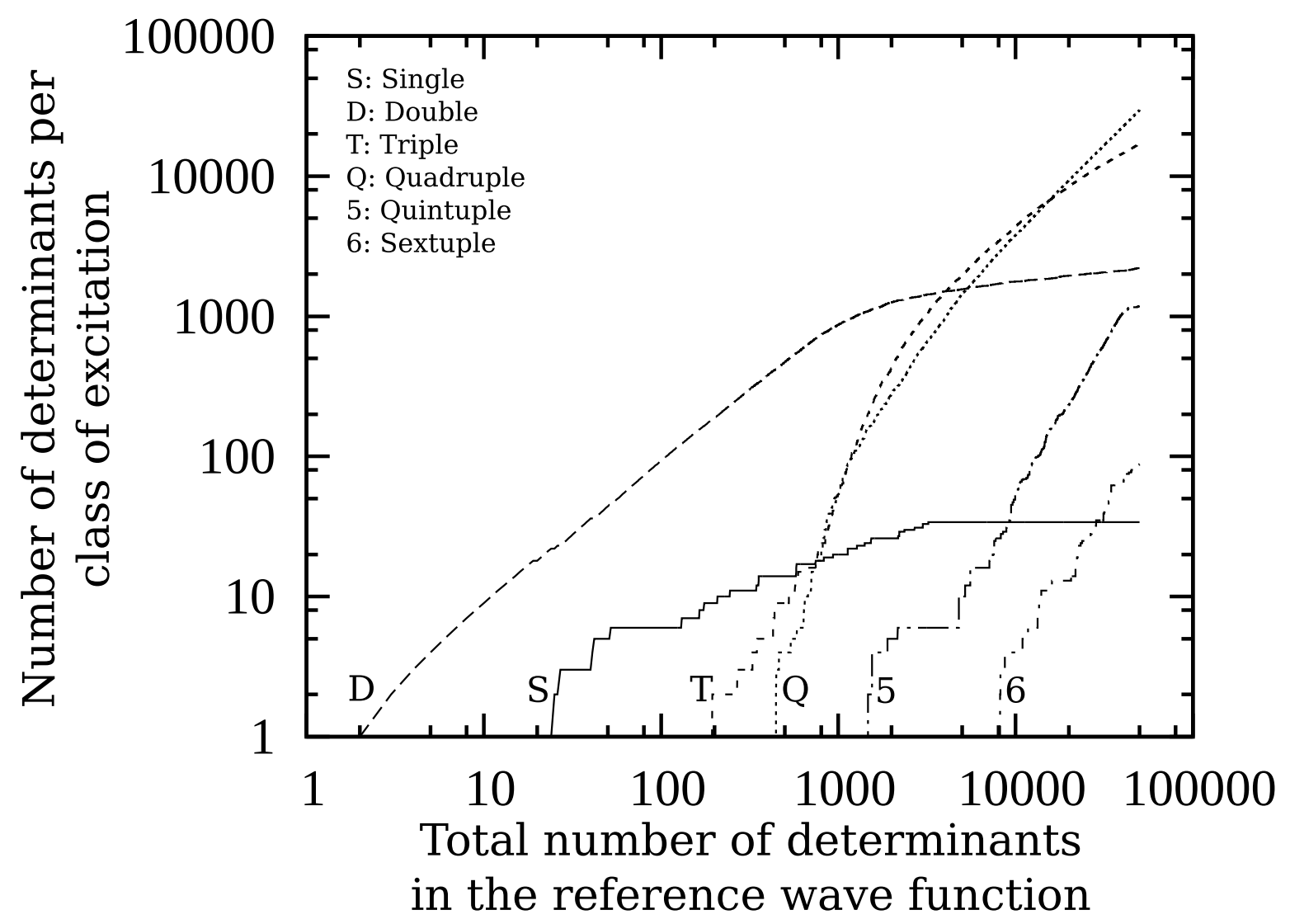

FIG. 3: 


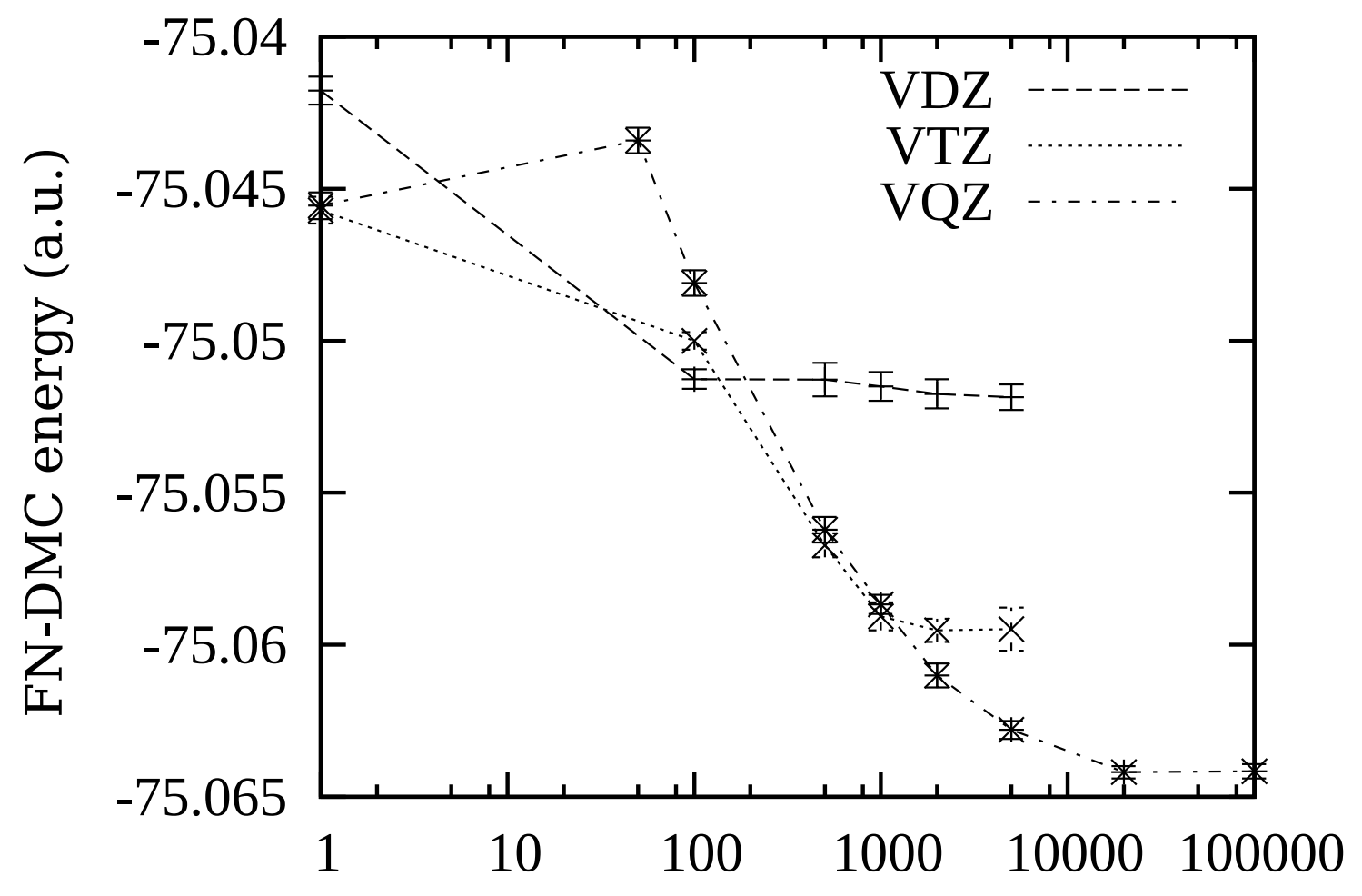

Number of determinants in the reference wave function

FIG. 4: 


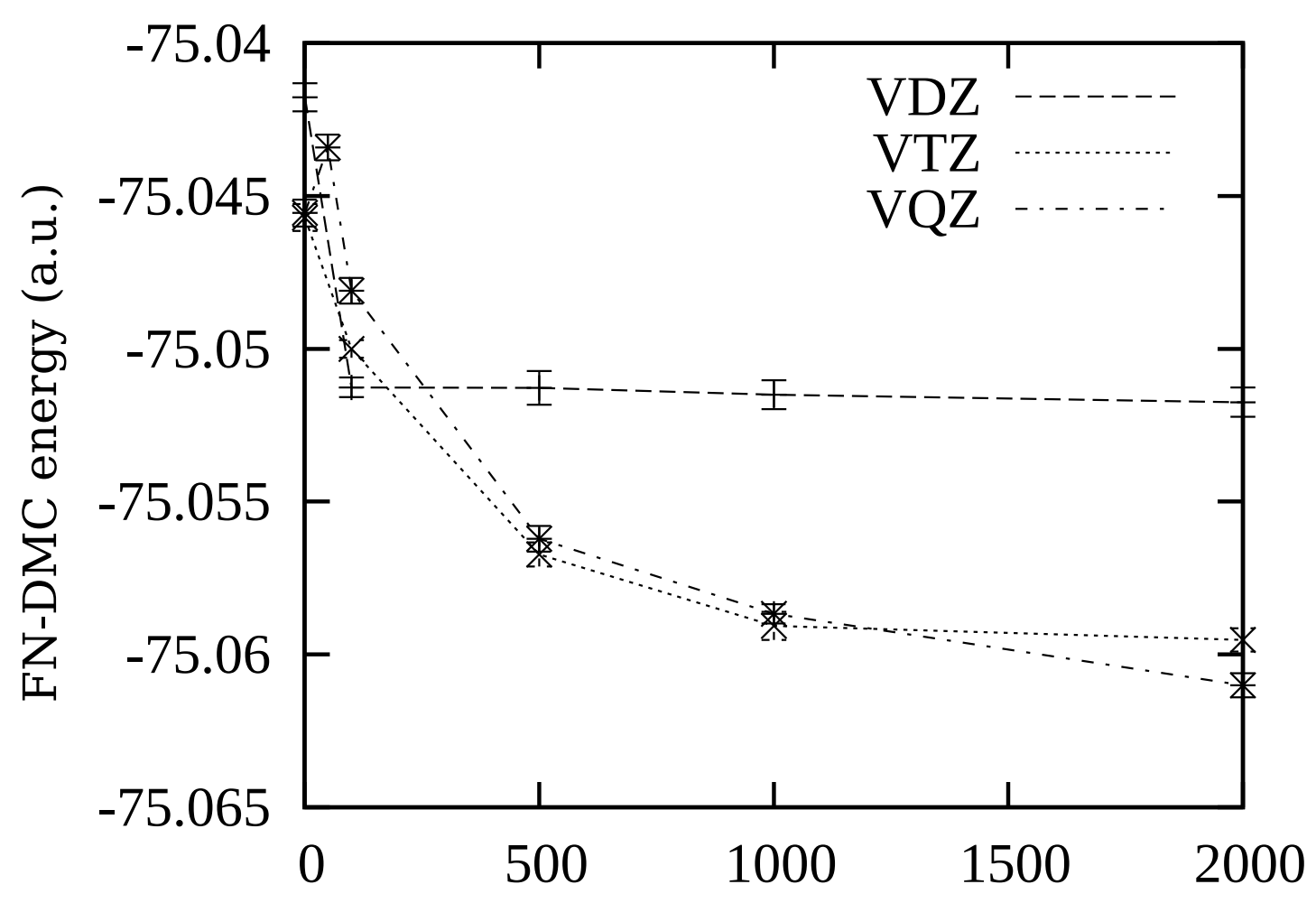

Number of determinants in the reference wave function

FIG. 5: 